\title{
Society of Biomolecular Imaging and Informatics Special Issue
}

\author{
Myles Fennell \\ Guest Editor \\ Memorial Sloan-Kettering Cancer Center, USA. \\ Kaylene J. Simpson \\ Guest Editor \\ Peter MacCallum Cancer Centre, Australia.
}

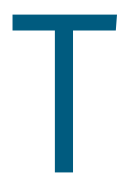

he Society of Biomolecular Imaging and Informatics ( $\mathrm{SBI}^{2}$; www.sbi2.org) is an international community of leaders, scientists, and students promoting technological advancement, discovery, and education to interrogate biological models quantitatively in order to provide high-context information at the cellular level. The Society was established in 2012 and delivered its 1st annual conference in September 2014. The 2nd annual $\mathrm{SBI}^{2}$ conference will be held September 15-17, 2015, at the Joseph B. Martin Conference Center at Harvard Medical School in Boston, MA. The goal of $\mathrm{SBI}^{2}$ is to offer the scientific community a high-value, low-cost meeting with experts in the fields of high-content screening (HCS) and high-content analysis (HCA) from across the globe. This 3-day meeting will offer short courses in HCA/ HCS, networking opportunities, and a well-rounded scientific program with a focus on recent research.

The conference will open with elective training sessions on a diverse series of topics from assay development and screening, to image analysis, to tissue-based HCS. These will be followed by a guided roundtable and colloquium on best practices and unmet needs HCS/HCA, designed for more experienced users. The colloquium will cover multiple topics, led by eminent discussion leaders, covering aspects of image and data analysis for 2D and 3D biology that will provide users with the tools and expertise to translate complex biology into successful screening campaigns.

A full 2 days of scientific presentations, technology spotlights, and social events will kick off with a keynote presentation from Dr. Lani Wu from UCSF on "Selecting optimal biomarkers for phenotypic screening." Scientific sessions will cover HCA assay development and implementation, image analysis and informatics, phenotypic drug discovery, and innovative imaging. Dr. Sangeeta Bhatia from the Broad In- stitute of MIT and Harvard will give the closing keynote presentation on "Engineered livers for modeling health."

A special issue of ASSAY and Drug Development Technologies was published to coincide with the $1 \mathrm{st} \mathrm{SBI}^{2}$ annual meeting, and $\mathrm{SBI}^{2}$ has teamed up once again with ASSAY to publish two special issues: this one to be made available at the September $\mathrm{SBI}^{2}$ conference, and a second to follow shortly thereafter. These issues comprise research papers on HCS and HCA covering the wide array of scientific interests, technologies, and therapeutic areas important to $\mathrm{SBI}^{2}$ members and the wider HCS/HCA community. In addition, this first issue includes abstracts from the invited speakers in each session; the poster abstracts and the remaining podium abstracts will be published in the second issue.

This first special issue opens with a commentary by 0 . Joseph Trask and Paul Johnston, past and current presidents of $\mathrm{SBI}^{2}$, discussing the important issue of data and material standards, highlighting areas in which $\mathrm{SBI}^{2}$ are taking the lead within the field to drive change and compliance. The six research articles include four discovery projects, each targeting a specific phenotype-two in oncology, one in neuronal regeneration, and one in parasitic infection. The other two papers describe methods that will be useful in a broad range of studies. In oncology, the work by Fennell et al. covers a full genome siRNA screen to discover novel therapeutic targets for mutant HRAS expressing tumors by preventing them from receiving increased nutrients through macropinocytosis. This method was used to identify novel modulators as well as novel targets through quantification of a fluorescently tagged dextran and then using both intensity and cellular localization of macropinosome vesicles. Johnston et al. report on a high-throughput compound screen of almost 100,000 compounds for the inhibition of STAT-3 activation in IL-6 stimulated head and neck tumor cell lines. Increased STAT3 activation is linked to a poor prognosis, and limited therapeutic options are available.

In the neuroscience drug discovery area, Beckerman et al. describe an imaging-based assay for the reactive activation of astrocytes in response to treatment with kinase inhibitors. This is a counter-screening assay for kinase inhibitors already identified as having positive effects on neuronal network 


\section{EDITORIAL}

recovery following traumatic spinal cord injury. To address a major parasitic disease, Dagley et al. present an HCS assay to screen for compounds that block the infection of THP-1 macrophages by the Leishmania parasite. This method uses fluorescent dyes to stain both macrophage and parasite, without the need for creating recombinant cell lines for screening, and is therefore flexible in screening Leishmania strains or mutant macrophage with relative ease.

The final two papers describe broadly applicable HCA methods. Two areas gaining increasing attention are the development of imaging assays to quantitate 3-dimensional spheroid structures, and systems biology approaches to determine compound mechanisms of action. Sirenko et al. report a high-throughput approach to imaging human cancer spheroids in response to compound treatment. They quantitate a range of parameters, including spheroid size and shape and cell viability. Reisen et al. screened a set of annotated compounds and established a set of "HCS fingerprints," phenotypic parameters identified in six different cellular compartments that allow clustering of different novel compounds with known signaling pathways.

The second $\mathrm{SBI}^{2}$ special issue will feature a further selection of research articles and oral and poster abstracts from the September $\mathrm{SBI}^{2}$ conference. Detailed coverage of the advanced roundtable colloquium from day 1 of the meeting will follow at a later date. Together, the two issues will provide a focus for the $\mathrm{SBI}^{2}$ community worldwide, as well as much that we hope will be of interest to the broader readership of ASSAY. 